\title{
Nonresonant smoothing \\ for coupled wave + transport equations \\ and the Vlasov-Maxwell system
}

\section{François Bouchut, François Golse and Christophe Pallard}

\begin{abstract}
Consider a system consisting of a linear wave equation coupled to a transport equation:

$$
\begin{aligned}
\square_{t, x} u & =f, \\
\left(\partial_{t}+v(\xi) \cdot \nabla_{x}\right) f & =P\left(t, x, \xi, D_{\xi}\right) g,
\end{aligned}
$$

Such a system is called nonresonant when the maximum speed for particles governed by the transport equation is less than the propagation speed in the wave equation. Velocity averages of solutions to such nonresonant coupled systems are shown to be more regular than those of either the wave or the transport equation alone. This smoothing mechanism is reminiscent of the proof of existence and uniqueness of $C^{1}$ solutions of the Vlasov-Maxwell system by R. Glassey and W. Strauss for time intervals on which particle momenta remain uniformly bounded, in "Singularity formation in a collisionless plasma could occur only at high velocities", Arch. Rational Mech. Anal. 92 (1986), no. 1, 59-90. Applications of our smoothing results to solutions of the Vlasov-Maxwell system are discussed.
\end{abstract}

\section{Nonresonant coupled wave + transport systems}

Consider a coupled system consisting of a linear wave equation and a transport equation, of the form

$$
\begin{aligned}
\square_{t, x} u & =f, \\
\left(\partial_{t}+v(\xi) \cdot \nabla_{x}\right) f & =P\left(t, x, \xi, D_{\xi}\right) g
\end{aligned}
$$

where $\square_{t, x}=\partial_{t}^{2}-\Delta_{x}$.

2000 Mathematics Subject Classification: 35B65, 35B34, 35L05, 35Q75, 82C40, 82D10. Keywords: Wave equation, transport equation, velocity averaging, Vlasov-Maxwell system. 
The unknowns in that system are the real-valued functions $u \equiv u(t, x, \xi)$ and $f \equiv f(t, x, \xi)$, while the source term in the right-hand side of the transport equation involves a given real-valued function $g \equiv g(t, x, \xi)$. The notation $P\left(t, x, \xi, D_{\xi}\right)$ designates a (smooth) linear differential operator in the variable $\xi$ only, while $v \equiv v(\xi)$ is a smooth $\mathbb{R}^{D}$-valued vector field on $\mathbb{R}^{M}$.

The system (1.1) is posed for all $(t, x, \xi) \in \mathbb{R}_{+}^{*} \times \mathbb{R}^{D} \times \mathbb{R}^{M}$. Associated to this system are the initial conditions

$$
\begin{aligned}
u_{\mid t=0} & =u_{I}, \\
\partial_{t} u_{\mid t=0} & =u_{I}^{\prime}, \\
f_{\mid t=0} & =f_{I},
\end{aligned}
$$

where the functions $u_{I}, u_{I}^{\prime}, f_{I}$, together with $g$, are the data of the Cauchy problem (1.1)-(1.2).

The subject matter of this work is the local regularity of averages with respect to $\xi$ of the unknown $u$, namely of functions of the form

$$
\rho_{\chi} \equiv \rho_{\chi}(t, x)=\int u(t, x, \xi) \chi(\xi) d \xi
$$

where $\chi$ is an arbitrary test function in $C_{c}^{\infty}\left(\mathbb{R}^{M}\right)$.

One possible approach to this problem would be

- to first establish the regularity of velocity averages of the solution $f$ of the transport equation

$$
\int f(t, x, \xi) \chi(\xi) d \xi
$$

- and since averaging in $\xi$ commutes with the d'Alembert $\square_{t, x}$ operator, to infer the regularity of $\rho_{\chi}$ from the classical energy estimate for the wave equation

$$
\square_{t, x} \rho_{\chi}=\int f \chi d \xi
$$

the regularity of its right hand side obtained at the previous step and that of the initial data $u_{I}, u_{I}^{\prime}$.

Step 1 in this procedure is by now classical in kinetic theory: for smooth, generic $v$ 's,

$$
\int f \chi d \xi \in H_{l o c}^{\frac{1}{2(m+1)}}\left(\mathbb{R}_{+} \times \mathbb{R}^{D}\right) \text { if } g \text { and } f \in L_{l o c}^{2}\left(\mathbb{R}_{+} \times \mathbb{R}^{D} \times \mathbb{R}^{M}\right),
$$

where $m$ is the order of the differential operator $P\left(t, x, \xi, D_{\xi}\right)$ involved in the right-hand side of the transport equation in (1.1). 
This gain of regularity was observed for the first time in [11], [12] for $m=0$ and [5] for $m \in \mathbb{N}^{*}$ and is referred to as smoothing by Velocity Averaging. The precise condition on a smooth vector field $v$ required for (1.4) to hold is that

(VA)

$$
\sup _{\epsilon>0} \sup _{(\omega, k) \in \mathbb{R} \times \mathbb{R}^{D}} \frac{1}{\epsilon} \text { meas }\left(\left\{\xi \in \operatorname{supp} \chi|| \omega+v(\xi) \cdot k \mid \leq \epsilon \sqrt{\omega^{2}+|k|^{2}}\right\}\right)<+\infty .
$$

The classical energy estimate for the wave equation (see [13, formula (6.3.1)]) finally implies that

$$
\rho_{\chi} \in H_{l o c}^{1+\frac{1}{2(m+1)}}\left(\mathbb{R}_{+} \times \mathbb{R}^{D}\right) \text { if } g \text { and } f \in L_{l o c}^{2}\left(\mathbb{R}_{+} \times \mathbb{R}^{D} \times \mathbb{R}^{M}\right),
$$

provided that

$$
u_{I} \in L^{2}\left(\mathbb{R}^{M} ; H_{l o c}^{1+\frac{1}{2(m+1)}}\left(\mathbb{R}_{+} \times \mathbb{R}^{D}\right)\right), \quad u_{I}^{\prime} \in L^{2}\left(\mathbb{R}^{M} ; H_{l o c}^{\frac{1}{2(m+1)}}\left(\mathbb{R}_{+} \times \mathbb{R}^{D}\right)\right) .
$$

However, this method fails to predict the exact amount of regularity on $\rho_{\chi}$ for a large class of systems (1.1), namely those for which

$$
v_{M}:=\sup _{\xi \in \operatorname{supp} \chi}|v(\xi)|<1 .
$$

The relevance of this condition comes from physical considerations. Various kinetic models describe the coupling of particle transport with a background electromagnetic field. For massive particles with uniformly bounded momenta (i.e. with momenta in the support of $\chi$ ), the maximum speed of transport is less than the speed of light (normalized here to 1), with a uniform bound as in (NR).

In order to gain some intuition on the role of this non-resonance condition (NR) in the regularity problem for $\rho_{\chi}$ as in (1.3), we propose the following line of reasoning in the case where $P\left(t, x, \xi, D_{\xi}\right)$ is the identity (or equivalently, $m=0$ ). To avoid unnecessary complications, we also assume that the initial conditions $u_{I}$ and $u_{I}^{\prime}$ are smooth.

Under assumption (NR), the characteristic manifold of the wave operator

$$
\text { Char }\left(\square_{t, x}\right)=\left\{\left.(t, x, \omega, k) \in T^{*}\left(\mathbb{R}_{+}^{*} \times \mathbb{R}^{D}\right)\left|\omega^{2}-\right| k\right|^{2}=0\right\}
$$

and that of the transport operator

$$
\text { Char }\left(\partial_{t}+v(\xi) \cdot \nabla_{x}\right)=\left\{(t, x, \omega, k) \in T^{*}\left(\mathbb{R}_{+}^{*} \times \mathbb{R}^{D}\right) \mid \omega+v(\xi) \cdot k=0\right\}
$$

intersect at the zero section:

Char $\left(\square_{t, x}\right) \cap$ Char $\left(\partial_{t}+v(\xi) \cdot \nabla_{x}\right)=\left\{(t, x, 0,0) \mid t>0, x \in \mathbb{R}^{D}\right\}$. 
Consider a point $\left(t_{0}, x_{0}, \omega, k\right) \in T^{*}\left(\mathbb{R}_{+}^{*} \times \mathbb{R}^{D}\right)$ such that $(\omega, k) \neq(0,0)$. Then

- either $\left(t_{0}, x_{0}, \omega, k\right) \notin$ Char $\left(\square_{t, x}\right)$, and thus $u$ has two derivatives more than $f$ microlocally at point $\left(t_{0}, x_{0}, \omega, k\right)$;

- or $\left(t_{0}, x_{0}, \omega, k\right) \notin$ Char $\left(\partial_{t}+v(\xi) \cdot \nabla_{x}\right)$, and thus $u$ has one derivative more than $w=\left(\partial_{t}+v(\xi) \cdot \nabla_{x}\right) u$ microlocally at point $\left(t_{0}, x_{0}, \omega, k\right)$; then in the scale of $L^{2}$-based Sobolev spaces, $w=\left(\partial_{t}+v(\xi) \cdot \nabla_{x}\right) u$ has one derivative more than $g$ at $\left(t_{0}, x_{0}, \omega, k\right)$, independently of whether this point belongs to Char $\left(\square_{t, x}\right)$ or not, by the usual energy estimate for the wave equation $\square_{t, x} w=g$ satisfied by $w$.

This little argument suggests that, for an arbitrary fixed $\xi$, if the differential operator $P\left(t, x, \xi, D_{\xi}\right)=I d$ (or more generally is of order 0 ) and $|v(\xi)|<1$, then $u(\cdot, \cdot, \xi) \in H_{\text {loc }}^{2}\left(\mathbb{R}_{+} \times \mathbb{R}^{D}\right)$ as soon as ${ }^{1}$ both $f$ and $g \in$ $L_{\text {loc }}^{2}\left(\mathbb{R}_{+} \times \mathbb{R}^{D}\right)$.

This gain of regularity is not only better than (1.5) even in the case of $m=0$, but also relies on a completely different mechanism, as witnessed by the fact that this smoothing effect occurs pointwise in $\xi$. At variance, the former procedure relies fundamentally on smoothing the solution of the transport equation by averaging in $\xi$, as implied by condition (VA). It also completely separates the roles of both the transport and wave equations in (1.1), while the new mechanism for smoothing described above is based on the joint properties of the transport and wave equations in (1.1).

Below we call this mechanism "nonresonant smoothing" in view of its analogy with the classical envelope theory for the Mathieu equation (see [2, §17] or D. Pesme's contribution in [4]).

The microlocal argument above fails however to indicate what happens in the important case where the differential operator in the right hand side of (1.1) has order $m>0$; it also fails in the case where the regularity is measured in $L^{p}$-based Sobolev spaces for $1 \leq p \leq+\infty$, with $p \neq 2$ and for space dimension 3 . This is of course the most relevant case in view of physical applications (see the next section).

The outline of the paper is as follows: Section 2 states the main results on nonresonant smoothing, together with explicit counterexamples showing that our statements are sharp. Section 3 explains how the relativistic VlasovMaxwell (RVM) system can be put in the form (1.1). Section 4 discusses applications to the smoothness of solutions to the (RVM) system.

\footnotetext{
${ }^{1}$ An analogous important observation was communicated by S. Klainerman to the second author: under integration along a time-like curve, solutions of the wave equation gain in regularity relative to the space variables. (This is a natural amplification of Proposition 2.7 in [14].)
} 


\section{Nonresonant smoothing: main results}

As suggested in the previous section, the most direct way to measure nonresonant smoothing is in $L^{2}$-based Sobolev spaces. Indeed these are natural spaces for the classical energy estimate of the wave equation.

Theorem 1 Let $f$ and $g \in L_{\text {loc }}^{2}\left(\mathbb{R}_{+}^{*} \times \mathbb{R}^{D} \times \mathbb{R}^{M}\right)$, and assume that the initial data $f_{I} \in L_{\text {loc }}^{2}\left(\mathbb{R}^{D} \times \mathbb{R}^{M}\right)$, that $u_{I}^{\prime} \in L_{\text {loc }}^{2}\left(\mathbb{R}_{\xi}^{M} ; H_{\text {loc }}^{1}\left(\mathbb{R}^{D}\right)\right)$ while $u_{I} \in$ $L_{\text {loc }}^{2}\left(\mathbb{R}_{\xi}^{M} ; H_{\text {loc }}^{2}\left(\mathbb{R}^{D}\right)\right)$. Let $P\left(t, x, \xi, D_{\xi}\right)$ be a linear differential operator of order $m \in \mathbb{N}$ on $\mathbb{R}_{\xi}^{M}$ with smooth coefficients. Pick $\chi \equiv \chi(\xi)$ be a test function in $C_{c}^{m}\left(\mathbb{R}^{M}\right)$ and let $v \equiv v(\xi)$ be in $C^{m}\left(\mathbb{R}^{M}\right)$ and satisfy the nonresonant condition $(N R)$.

Then, if (1.1)-(1.2) hold, the $\xi$-average

$$
\rho_{\chi}(t, x)=\int u(t, x, \xi) \chi(\xi) d \xi
$$

belongs to $H_{\text {loc }}^{2}\left(\mathbb{R}_{+}^{*} \times \mathbb{R}^{D}\right)$.

There is an analogous statement in space dimension 3 , with $L^{2}$ and $H^{s}$ replaced by $L^{p}$ and $W^{s, p}$, for $1<p<2$ and $2<p<\infty$. The proof is essentially the same as in the $L^{2}$ case, except for the arguments that involve the energy estimate for the wave equation. These are replaced by the fact that the elementary solution of the wave operator expressing $u$ in terms of $f$ is proportional to the uniform measure on the unit sphere $S^{2}$, to which one can apply the corollary to theorem 7 of [6]. One eventually finds that

$$
\rho_{\chi} \in W_{l o c}^{s, p}\left(\mathbb{R}_{+}^{*} \times \mathbb{R}^{D}\right), \quad \text { where } \quad s=1+2 \inf \left(\frac{1}{p}, \frac{1}{p^{\prime}}\right) .
$$

Because this approach relies in the end on $L^{p}$ estimates for the elliptic operator $Q_{\xi}^{\lambda}$, the cases $p=1$ or $p=+\infty$ require a different treatment based on the commutation of the Lorentz boosts $L_{j}=x_{j} \partial_{t}+t \partial_{x_{j}}, j=1,2,3$ with $\square_{t, x}$ : this part bears some definite analogy with one of the key techniques in [8].

Proof of Theorem 1. First observe that if $f$ and $f_{I}$ are null functions, then the transport part in system (1.1) vanishes and theorem 1 follows from the regularity properties of the wave operator $\square_{t, x}$. By linearity we now assume $u_{I}=u_{I}^{\prime}=0$.

The key argument in the proof of theorem 1 is that some well chosen combinations of the wave operator $\square_{t, x}$ and of the transport operators

$$
T^{ \pm}=\partial_{t} \pm v(\xi) \cdot \nabla_{x}
$$

are elliptic in the variables $t$ and $x$. 
Lemma 1 For $\chi \in C_{c}^{m}\left(\mathbb{R}^{M}\right)$, let $v \equiv v(\xi)$ in $C^{m}\left(\mathbb{R}^{M}\right)$ satisfy the nonresonant condition $(N R)$, and let $\lambda \in \mathbb{R}$. The two following conditions are equivalent:

- $\lambda$ satisfies the condition

$$
v_{M}^{2}<\lambda<1, \quad \text { where } v_{M}=\sup _{\xi \in \operatorname{supp} \chi}|v(\xi)|
$$

- for each $\xi \in \operatorname{supp} \chi$, the second order differential operator

$$
Q_{\xi}^{\lambda}=\lambda \square_{t, x}-\left(\partial_{t}-v(\xi) \cdot \nabla_{x}\right)\left(\partial_{t}+v(\xi) \cdot \nabla_{x}\right)
$$

is elliptic.

When $\lambda$ verifies any of these conditions, the symbol $q_{\xi}^{\lambda}$ of the operator $Q_{\xi}^{\lambda}$ satisfies the following uniform ellipticity estimates: for all $m \in \mathbb{N}$

$$
\sup _{\xi \in \operatorname{supp} \chi} \sup _{\omega^{2}+|k|^{2}>0}\left(\omega^{2}+|k|^{2}\right)\left|D_{\xi}^{m}\left(\frac{1}{q_{\xi}^{\lambda}(\omega, k)}\right)\right|<+\infty .
$$

The uniform ellipticity estimates (2.3) provide precisely the quantitative information missing in the little microlocal argument of the previous section and necessary to address the case where the source term of the transport equation in (1.1) effectively involves $\xi$ derivatives. Notice that one could also use the operator $\lambda \square_{t, x}-\left(\partial_{t}+v(\xi) \cdot \nabla_{x}\right)^{2}$ instead of $Q_{\xi}^{\lambda}$.

Proof of Lemma 1. The symbol $q_{\xi}^{\lambda}(\omega, k)=\lambda\left(-\omega^{2}+|k|^{2}\right)+(\omega-v \cdot k)(\omega+v \cdot k)$ is a homogeneous function of order 2 of the Fourier variables $(\omega, k)$. Notice that a $\xi$ derivative does not affect this property, so that:

$$
D_{\xi}^{m}\left(\frac{1}{q_{\xi}^{\lambda}(\omega, k)}\right)=\frac{N_{\xi}^{\lambda}(\omega, k)}{q_{\xi}^{\lambda}(\omega, k)^{2^{m}}}
$$

with $N_{\xi}^{\lambda}(\omega, k)$ homogeneous of order $2^{m+1}-2$. Hence

$$
\sup _{\omega^{2}+|k|^{2}>0}\left(\omega^{2}+|k|^{2}\right)\left|D_{\xi}^{m}\left(\frac{1}{q_{\xi}^{\lambda}(\omega, k)}\right)\right|=\sup _{\omega^{2}+|k|^{2}=1}\left|D_{\xi}^{m}\left(\frac{1}{q_{\xi}^{\lambda}(\omega, k)}\right)\right| .
$$

Besides, the Cauchy-Schwarz inequality implies the following lower bound for $q_{\xi}^{\lambda}(\omega, k)$ :

$$
(1-\lambda) \omega^{2}+\lambda|k|^{2}-(v \cdot k)^{2} \geq(1-\lambda) \omega^{2}+\lambda|k|^{2}-|v|^{2}|k|^{2} .
$$


If (2.1) holds, then

$$
m_{\lambda}=\min \left(1-\lambda, \inf _{\xi \in \operatorname{supp} \chi}\left(\lambda-|v(\xi)|^{2}\right)\right)>0 .
$$

Therefore $q_{\xi}^{\lambda}(\omega, k) \geq m_{\lambda}\left(\omega^{2}+|k|^{2}\right)$, and (2.4) gives:

$$
\sup _{\omega^{2}+|k|^{2}>0}\left(\omega^{2}+|k|^{2}\right)\left|D_{\xi}^{m}\left(\frac{1}{q_{\xi}^{\lambda}(\omega, k)}\right)\right| \leq \frac{1}{m_{\lambda}^{2^{m}}} \sup _{\omega^{2}+|k|^{2}=1}\left|N_{\xi}^{\lambda}(\omega, k)\right| .
$$

Since the right hand side of the inequality above depends continuously on $\xi$, we infer the result (2.3) for any compactly supported function $\chi$. Conversely, when (2.1) is not satisfied, it is obvious that the operator $Q_{\xi}^{\lambda}$ is not elliptic for some $\xi \in \operatorname{supp} \chi$.

Once lemma 1 is established, the proof of theorem 1 is based upon controlling $Q_{\xi}^{\lambda} u$ by the usual energy estimate for the wave equation. Finally, the uniform ellipticity estimates (2.3) are used to control the various contributions to the $\xi$-average $\rho_{\chi}$ after integrating by parts to bring all $\xi$-derivatives to bear on either $\chi$ or $1 / q_{\xi}^{\lambda}$. We summarize in lemma 2 some facts about the inhomogeneous wave equation in (1.1). Detailed results for the wave operator may be found in [17].

Lemma 2 Consider the Cauchy problem:

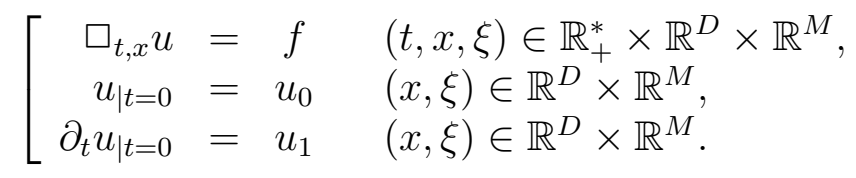

where $f \in L_{\text {loc }}^{2}\left(\mathbb{R}_{t}^{+} \times \mathbb{R}_{x}^{D} \times \mathbb{R}_{\xi}^{M}\right)$ and with initial data

$$
u_{0} \in L_{l o c}^{2}\left(\mathbb{R}_{\xi}^{M} ; H_{l o c}^{1}\left(\mathbb{R}_{x}^{D}\right)\right) \quad \text { and } \quad u_{1} \in L_{l o c}^{2}\left(\mathbb{R}_{\xi}^{M} ; L_{l o c}^{2}\left(\mathbb{R}_{x}^{D}\right)\right) .
$$

Then there exists a solution $u$ to (2.5) such that for almost every $\xi \in \mathbb{R}^{M}$,

$$
u(\cdot, \cdot, \xi) \in \mathcal{C}\left([0, T], H_{l o c}^{1}\left(\mathbb{R}^{D}\right)\right) \cap \mathcal{C}^{1}\left([0, T], L_{l o c}^{2}\left(\mathbb{R}^{D}\right)\right) .
$$

Moreover this solution satisfies $u \in L_{\text {loc }}^{2}\left(\mathbb{R}_{\xi}^{M} ; H_{\text {loc }}^{1}\left(\mathbb{R}_{+}^{*} \times \mathbb{R}^{D}\right)\right)$.

For an arbitrary $\lambda$, we have:

$$
Q_{\xi}^{\lambda} u=\lambda \square_{t, x} u-T_{\xi}^{-} T_{\xi}^{+} u
$$

The wave equation in (1.1) gives $\lambda \square_{t, x} u=\lambda f$. Now if we merge the two relations in the system (1.1), we get: 
Lemma 3 Suppose that $(u, f, g)$ satisfy (1.1) with null initial conditions on $u$. We note $P\left(t, x, \xi, D_{\xi}\right) \phi=\sum_{|\alpha| \leq m} \partial_{\xi}^{\alpha}\left(a_{\alpha}(t, x, \xi) \phi\right)$, and define $h_{\alpha}$ as the solution of the Cauchy problem

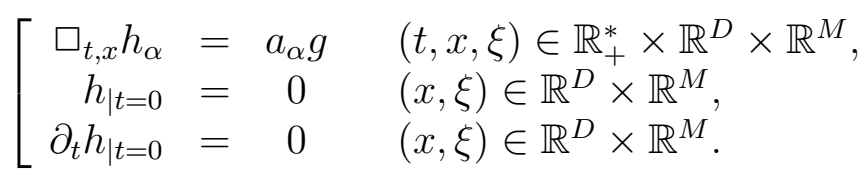

Define also $h^{I}$ as the solution of

$$
\left[\begin{array}{rlll}
\square_{t, x} h^{I} & =0 & & (t, x, \xi) \in \mathbb{R}_{+}^{*} \times \mathbb{R}^{D} \times \mathbb{R}^{M} \\
h_{\mid t=0} & =0 & & (x, \xi) \in \mathbb{R}^{D} \times \mathbb{R}^{M} \\
\partial_{t} h_{\mid t=0} & =f_{I} & & (x, \xi) \in \mathbb{R}^{D} \times \mathbb{R}^{M}
\end{array}\right.
$$

Then we have $T_{\xi}^{+} u=\sum_{|\alpha| \leq m} \partial_{\xi}^{\alpha} h_{\alpha}+h^{I}$.

Proof of Lemma 3. The existence of the functions $h_{\alpha}$ and $h^{I}$ is a consequence of lemma 2. Consider $\sum_{|\alpha| \leq m} \partial_{\xi}^{\alpha} h_{\alpha}+h^{I}$ and $T_{\xi}^{+} u$. The definition of $h_{\alpha}$ implies that

$$
\square_{t, x}\left(\sum_{|\alpha| \leq m} \partial_{\xi}^{\alpha} h_{\alpha}\right)=\sum_{|\alpha| \leq m} \partial_{\xi}^{\alpha} \square_{t, x} h_{\alpha}=\sum_{|\alpha| \leq m} \partial_{\xi}^{\alpha}\left(a_{\alpha} g\right)=P\left(t, x, \xi, D_{\xi}\right) g .
$$

Therefore,

$$
\square_{t, x}\left(\sum_{|\alpha| \leq m} \partial_{\xi}^{\alpha} h_{\alpha}+h^{I}\right)=P\left(t, x, \xi, D_{\xi}\right) g .
$$

The relations in (1.1) gives:

$$
\square_{t, x} T_{\xi}^{+} u=T_{\xi}^{+} \square_{t, x} u=T_{\xi}^{+} f=P\left(t, x, \xi, D_{\xi}\right) g .
$$

The initial conditions are satisfied:

$$
\left(\sum_{|\alpha| \leq m} \partial_{\xi}^{\alpha} h_{\alpha}+h^{I}\right)_{\mid t=0}=0 \text { and } \partial_{t}\left(\sum_{|\alpha| \leq m} \partial_{\xi}^{\alpha} h_{\alpha}+h^{I}\right)_{\mid t=0}=f_{I} .
$$

Similarly, we have for $T_{\xi}^{+} u$ :

$$
\begin{aligned}
\left(T_{\xi}^{+} u\right)_{\mid t=0} & =\partial_{t} u_{\mid t=0}+v(\xi) \cdot \nabla_{x} u_{\mid t=0}=0, \\
\partial_{t}\left(T_{\xi}^{+} u\right)_{\mid t=0} & =\left(f+\triangle_{x} u\right)_{\mid t=0}+v(\xi) \cdot \nabla_{x} u_{\mid t=0}=f_{I} .
\end{aligned}
$$

Since $\sum_{|\alpha| \leq m} \partial_{\xi}^{\alpha} h_{\alpha}+h^{I}$ and $T_{\xi}^{+} u$ solve the same Cauchy problem, they must coincide everywhere. 
It follows from lemma 3 that $Q_{\xi}^{\lambda} u=\lambda f-\sum_{|\alpha| \leq m} T_{\xi}^{-} \partial_{\xi}^{\alpha} h_{\alpha}-T_{\xi}^{-} h^{I}$. We write:

$$
Q_{\xi}^{\lambda} u=\lambda f-\sum_{|\alpha| \leq m}\left[T_{\xi}^{-}, \partial_{\xi}^{\alpha}\right] h_{\alpha}-\sum_{|\alpha| \leq m} \partial_{\xi}^{\alpha} T_{\xi}^{-} h_{\alpha}-T_{\xi}^{-} h^{I}
$$

We now localize $u$ with a test function $\phi \in \mathcal{C}_{c}^{\infty}\left(\mathbb{R}_{+}^{*} \times \mathbb{R}_{x}^{D}\right)$ :

$$
Q_{\xi}^{\lambda}(\phi u)=\phi Q_{\xi}^{\lambda} u+R_{\xi} u
$$

here $R_{\xi}$ is a first order linear differential operator in the $(t, x)$ variables, with smooth and compactly supported coefficients:

$$
Q_{\xi}^{\lambda}(\phi u)=\lambda \phi f-\phi \sum_{|\alpha| \leq m}\left[T_{\xi}^{-}, \partial_{\xi}^{\alpha}\right] h_{\alpha}-\phi \sum_{|\alpha| \leq m} \partial_{\xi}^{\alpha} T_{\xi}^{-} h_{\alpha}-\phi T_{\xi}^{-} h^{I}+R_{\xi} u \text {. }
$$

From lemma 2, we know that the first, second, fourth and last terms of the right hand side belong to $L_{\text {loc }}^{2}\left(\mathbb{R}^{M} ; L^{2}\left(\mathbb{R}_{+} \times \mathbb{R}^{D}\right)\right)$. Define $a_{\lambda}$ by:

$$
a_{\lambda}=\lambda \phi f-\phi \sum_{|\alpha| \leq m}\left[T_{\xi}^{-}, \partial_{\xi}^{\alpha}\right] h_{\alpha}-\phi T_{\xi}^{-} h^{I}+R_{\xi} u
$$

We get:

$$
Q_{\xi}^{\lambda}(\phi u)=a_{\lambda}-\phi \sum_{|\alpha| \leq m} \partial_{\xi}^{\alpha} T_{\xi}^{-} h_{\alpha}
$$

We apply the Fourier transform in the variables $(t, x)$ to the previous equality and denote by $(\omega, k)$ the corresponding Fourier variables.

$$
q_{\xi}^{\lambda} \widehat{\phi u}=\widehat{a_{\lambda}}-\sum_{|\alpha| \leq m} \partial_{\xi}^{\alpha}\left(\widehat{\phi T_{\xi}^{-} h_{\alpha}}\right)
$$

Now pick a test function $\chi \in \mathcal{C}_{c}^{\infty}\left(\mathbb{R}_{\xi}^{M}\right)$ and fix $\lambda$ such that condition (2.1) of lemma 1 holds. Averaging in $\xi$ in the sense of distributions, we find:

$$
\int \widehat{\phi u} \chi d \xi=\int \frac{1}{q_{\xi}^{\lambda}} \widehat{a_{\lambda}} \chi d \xi-\sum_{|\alpha| \leq m} \int \frac{1}{q_{\xi}^{\lambda}} \partial_{\xi}^{\alpha} \widehat{\phi T_{\xi}^{-} h_{\alpha}} \chi d \xi=I_{0}-\sum_{|\alpha| \leq m} I_{\alpha} .
$$

We want to establish that $\left(1+\omega^{2}+|k|^{2}\right) \int \widehat{\phi u} \chi d \xi$ belongs to $L_{\omega, k}^{2}$. But we already know $\int \widehat{\phi u} \chi d \xi \in L_{\omega, k}^{2}$ by lemma 2 , so it is enough to show that $\left(\omega^{2}+|k|^{2}\right) \int \widehat{\phi u} \chi d \xi \in L_{\omega, k}^{2}$. 
- Consider first

$$
I_{0} \equiv \int \frac{1}{q_{\xi}^{\lambda}} \widehat{a_{\lambda}} \chi d \xi
$$

By the Cauchy-Schwarz inequality,

$$
\left|\int \frac{1}{q_{\xi}^{\lambda}} \widehat{a_{\lambda}} \chi d \xi\right|^{2} \leq \int\left(\frac{\chi}{q_{\xi}^{\lambda}}\right)^{2} d \xi \int_{\operatorname{supp} \chi}\left|\widehat{a_{\lambda}}\right|^{2} d \xi .
$$

Obviously, $\mathbb{1}_{\operatorname{supp} \chi} a_{\lambda} \in L_{t, x, \xi}^{2}$ so $\mathbb{1}_{\operatorname{supp} \chi} \widehat{a_{\lambda}} \in L_{\omega, k, \xi}^{2}$ and

$$
\left\|\left(\omega^{2}+|k|^{2}\right) I_{0}\right\|_{L_{\omega, k}^{2}} \leq C_{0}(\lambda, \chi)\left\|1_{\operatorname{supp} \chi} a_{\lambda}\right\|_{L_{t, x, \xi}^{2}},
$$

with

$$
C_{0}(\lambda, \chi)=\left\|\int\left[\left(\omega^{2}+|k|^{2}\right) \frac{\chi}{q_{\xi}^{\lambda}}\right]^{2} d \xi\right\|_{L_{\omega, k}^{\infty}}^{\frac{1}{2}}
$$

- Now consider terms like

$$
I_{\alpha} \equiv \int \frac{1}{q_{\xi}^{\lambda}} \partial_{\xi}^{\alpha} \widehat{\phi T_{\xi}^{-} h_{\alpha}} \chi d \xi
$$

which can be written as:

$$
(-1)^{|\alpha|} \int \widehat{\phi T_{\xi}^{-} h_{\alpha}} \partial_{\xi}^{\alpha}\left(\frac{\chi}{q_{\xi}^{\lambda}}\right) d \xi
$$

after integrating by parts. We apply the Cauchy-Schwarz inequality again:

$$
\left|\int \widehat{\phi T_{\xi}^{-} h_{\alpha}} \partial_{\xi}^{\alpha}\left(\frac{\chi}{q_{\xi}^{\lambda}}\right) d \xi\right|^{2} \leq \int\left[\partial_{\xi}^{\alpha}\left(\frac{\chi}{q_{\xi}^{\lambda}}\right)\right]^{2} d \xi \int_{\operatorname{supp} \chi}\left|\widehat{\phi T_{\xi}^{-} h_{\alpha}}\right|^{2} d \xi .
$$

From lemma 2, we know that $1_{\operatorname{supp} \chi} \phi T_{\xi}^{-} h_{\alpha} \in L_{t, x, \xi}^{2}$ and

$$
\left\|\left(\omega^{2}+|k|^{2}\right) I_{\alpha}\right\|_{L_{\omega, k}^{2}} \leq C_{\alpha}(\lambda, \chi)\left\|1_{\operatorname{supp} \chi} \phi T_{\xi}^{-} h_{\alpha}\right\|_{L_{t, x, \xi}^{2}}
$$

with

$$
C_{\alpha}(\lambda, \chi)=\left\|\int\left[\left(\omega^{2}+|k|^{2}\right) \partial_{\xi}^{\alpha}\left(\frac{\chi}{q_{\xi}^{\lambda}}\right)\right]^{2} d \xi\right\|_{L_{\omega, k}^{\infty}}^{\frac{1}{2}}
$$

It remains to bound the quantities $C_{0}$ and $C_{\alpha}$. This follow immediately from the uniform estimates (2.3) of lemma 1. 
The result stated in theorem 1 is sharp, unlike that in (1.5). First, the $H^{2}$ regularity is optimal.

Proposition 1 There exists $u \in L^{2}\left(\mathbb{R}_{+}^{*} \times \mathbb{R} \times \mathbb{R}^{M}\right)$ satisfying:

$$
\begin{aligned}
\square_{t, x} u(t, x, \xi) & =f(t, x, \xi) \in L^{2}\left(\mathbb{R}_{+}^{*} \times \mathbb{R} \times \mathbb{R}^{M}\right), \\
\left(\partial_{t}+v \partial_{x}\right) f(t, x) & =g(t, x, \xi) \in L^{2}\left(\mathbb{R}_{+}^{*} \times \mathbb{R} \times \mathbb{R}^{M}\right),
\end{aligned}
$$

and such that

$$
\rho_{\chi}=\int u(t, x, \xi) \chi(\xi) d \xi \notin H_{l o c}^{2+\epsilon}\left(\mathbb{R}_{+}^{*} \times \mathbb{R}\right) .
$$

Proof. Consider $u$ of the form:

$$
\begin{aligned}
u: \mathbb{R} \times \mathbb{R} & \rightarrow \mathbb{R} \\
(t, x) & \mapsto \Psi(x-t) \Phi(x+t) .
\end{aligned}
$$

Write the Fourier transform of $u$ as

$$
\begin{aligned}
\widehat{u}(\omega, k) & =\iint \Psi(x-t) \Phi(x+t) e^{-i(t \omega+x k)} d t d x \\
& =\iint \Psi(v) \Phi(u) e^{-i\left(\frac{u-v}{2} \omega+\frac{u+v}{2} k\right)} \frac{d u d v}{2} \\
& =\frac{1}{2} \iint \Psi(v) \Phi(u) e^{-i\left(\frac{\omega+k}{2} u+\frac{k-\omega}{2} v\right)} d u d v \\
& =\frac{1}{2} \int \Phi(u) e^{-i \frac{\omega+k}{2} u} d u \int \Psi(v) e^{-i \frac{k-\omega}{2} v} d v \\
& =\frac{1}{2} \widehat{\Phi}\left(\frac{\omega+k}{2}\right) \widehat{\Psi}\left(\frac{k-\omega}{2}\right) .
\end{aligned}
$$

The Sobolev $H^{s}$ norm for $u$ is given by:

$$
\begin{aligned}
\|u\|_{H^{s}}^{2} & =\frac{1}{4} \iint\left(1+\omega^{2}+k^{2}\right)^{s}\left|\widehat{\Phi}\left(\frac{\omega+k}{2}\right)\right|^{2}\left|\widehat{\Psi}\left(\frac{k-\omega}{2}\right)\right|^{2} d \omega d k \\
& =\frac{1}{2} \iint\left(1+(\alpha-\beta)^{2}+(\alpha+\beta)^{2}\right)^{s}|\widehat{\Phi}(\alpha)|^{2}|\widehat{\Psi}(\beta)|^{2} d \alpha d \beta \\
& =\frac{1}{2} \iint\left(1+2 \alpha^{2}+2 \beta^{2}\right)^{s}|\widehat{\Phi}(\alpha)|^{2}|\widehat{\Psi}(\beta)|^{2} d \alpha d \beta .
\end{aligned}
$$

Pick $\epsilon>0$. Choose $\Phi=\Phi_{\epsilon}$ with compact support included in $\mathbb{R}_{+}^{*}$ such that $\Phi_{\epsilon} \in H^{2} \backslash H^{2+\epsilon}$. Take $\Psi \in C_{c}^{\infty}\left(\mathbb{R}_{-}^{*}\right)$. Then $u_{\epsilon}$ satisfies 
- $u_{\epsilon} \in H^{2}$ :

$$
\begin{aligned}
\left\|u_{\epsilon}\right\|_{H^{2}}^{2} & =\frac{1}{2} \iint\left(1+2 \alpha^{2}+2 \beta^{2}\right)^{2}\left|\widehat{\Phi}_{\epsilon}(\alpha)\right|^{2}|\widehat{\Psi}(\beta)|^{2} d \alpha d \beta \\
& \leq \frac{1}{2} \iint\left(1+2 \alpha^{2}\right)^{2}\left(1+2 \beta^{2}\right)^{2}\left|\widehat{\Phi}_{\epsilon}(\alpha)\right|^{2}|\widehat{\Psi}(\beta)|^{2} d \alpha d \beta \\
& \leq \frac{1}{2} \int\left(1+2 \beta^{2}\right)^{2}|\widehat{\Psi}(\beta)|^{2} d \beta \int\left(1+2 \alpha^{2}\right)^{2}\left|\widehat{\Phi}_{\epsilon}(\alpha)\right|^{2} d \alpha \\
& \leq 8\|\Psi\|_{H^{2}}^{2}\left\|\Phi_{\epsilon}\right\|_{H^{2}}^{2}<+\infty .
\end{aligned}
$$

- $u_{\epsilon} \notin H^{2+\epsilon}$ :

$$
\begin{aligned}
\left\|u_{\epsilon}\right\|_{H^{2+\epsilon}}^{2} & =\frac{1}{2} \iint\left(1+2 \alpha^{2}+2 \beta^{2}\right)^{2+\epsilon}\left|\widehat{\Phi}_{\epsilon}(\alpha)\right|^{2}|\widehat{\Psi}(\beta)|^{2} d \alpha d \beta \\
& \geq \frac{1}{2} \iint\left(1+2 \alpha^{2}\right)^{2+\epsilon}\left|\widehat{\Phi}_{\epsilon}(\alpha)\right|^{2}|\widehat{\Psi}(\beta)|^{2} d \alpha d \beta \\
& \geq \frac{1}{2} \int|\widehat{\Psi}(\beta)|^{2} d \beta \int\left(1+\alpha^{2}\right)^{2+\epsilon}\left|\widehat{\Phi}_{\epsilon}(\alpha)\right|^{2} d \alpha \\
& \geq \frac{1}{2}\|\Psi\|_{L^{2}}^{2}\left\|\Phi_{\epsilon}\right\|_{H^{2+\epsilon}}^{2}=+\infty
\end{aligned}
$$

Since $\Phi_{\epsilon} \in H^{2}(\mathbb{R})$ we obtain:

$$
f_{\epsilon}(t, x)=\square_{t, x} u_{\epsilon}(t, x)=-4 \Psi^{\prime}(x-t) \Phi_{\epsilon}^{\prime}(x+t) \in H^{1}\left(\mathbb{R}_{+}^{*} \times \mathbb{R}\right),
$$

and

$$
g_{\epsilon}(t, x)=\left(\partial_{t}+v \partial_{x}\right) f(t, x) \in L^{2}\left(\mathbb{R}_{+}^{*} \times \mathbb{R}\right) \quad(|v|<1) .
$$

From assumptions on supports of $\Phi_{\epsilon}$ and $\Psi$, we infer that supp $u$ is compact and included in $\mathbb{R}_{+}^{*} \times \mathbb{R}$, so that we have $u_{\mid t=0} \equiv 0$ and $\partial_{t} u_{\mid t=0} \equiv 0$. But $u_{\epsilon} \notin H^{2+\epsilon}\left(\mathbb{R}_{+}^{*} \times \mathbb{R}\right)$, which implies $u_{\epsilon} \notin H_{\text {loc }}^{2+\epsilon}\left(\mathbb{R}_{+}^{*} \times \mathbb{R}\right)$.

Second, the nonresonant condition (NR) cannot be dispensed with. Otherwise, $\rho_{\chi}$ is in general less regular than $H^{2}$, as shown by the following example.

Proposition 2 There exists $u \in L_{l o c}^{2}\left(\mathbb{R}_{+}^{*} \times \mathbb{R} \times \mathbb{R}^{M}\right)$ such that

$$
\begin{aligned}
\square_{t, x} u(t, x, \xi) & =f(t, x, \xi) \in L_{l o c}^{2}\left(\mathbb{R}_{+}^{*} \times \mathbb{R} \times \mathbb{R}^{M}\right), \\
\left(\partial_{t}-\partial_{x}\right) f(t, x) & =g(t, x, \xi) \in L_{l o c}^{2}\left(\mathbb{R}_{+}^{*} \times \mathbb{R} \times \mathbb{R}^{M}\right),
\end{aligned}
$$

but with

$$
\rho_{\chi}=\int u(t, x, \xi) \chi(\xi) d \xi \notin H_{l o c}^{2}\left(\mathbb{R}_{+}^{*} \times \mathbb{R}\right)
$$

Here $v(\xi) \equiv 1$. 
Proof. Consider

$$
u(x, t)=\Psi(x-t) \Phi(x+t),
$$

with $\Psi, \Phi$ to be chosen later. One has:

$$
\begin{aligned}
& \square_{t, x} u(t, x)=-4 \Psi^{\prime}(x-t) \Phi^{\prime}(x+t)=f(t, x), \\
& \left(\partial_{t}-\partial_{x}\right) f(t, x)=8 \Psi^{\prime \prime}(x-t) \Phi^{\prime}(x+t)=g(t, x) .
\end{aligned}
$$

The initial conditions are given by:

$$
u_{\mid t=0}=\Psi \Phi, \quad \partial_{t} u_{\mid t=0}=-\Psi^{\prime} \Phi+\Psi \Phi^{\prime} .
$$

Now pick $\Psi$ and $\Phi$ so that:

$$
\Psi \in C_{c}^{\infty}\left(\mathbb{R}_{-}^{*}\right), \quad \Phi(x)=x 1_{x>0} .
$$

Then $f$ and $g$ belong to $L_{l o c}^{2}\left(\mathbb{R} \times \mathbb{R}^{D}\right)$ :

$$
\begin{aligned}
& f(t, x, \xi)=-4 \Psi^{\prime}(x-t) \mathbb{1}_{x+t>0}, \\
& g(t, x, \xi)=8 \Psi^{\prime \prime}(x-t) \mathbb{1}_{x+t>0} .
\end{aligned}
$$

By construction, $u_{\mid t=0} \equiv 0$ and $\partial_{t} u_{\mid t=0} \equiv 0$. However $u \notin H_{l o c}^{2}\left(\mathbb{R} \times \mathbb{R}^{D}\right)$, for:

$$
\partial_{x}^{2} u=\partial_{x}^{2} \Psi^{-} \Phi^{+}+2 \partial_{x} \Psi^{-} \partial_{x} \Phi^{+}+\Psi^{-} \partial_{x}^{2} \Phi^{+}
$$

where $\Phi^{ \pm}(t, x)$ stands for $\Phi(x \pm t)$, and with the following derivatives in the sense of distributions:

$$
\begin{aligned}
\partial_{x} \Phi^{+}(t, x) & =1_{x+t>0}, \\
\partial_{x}^{2} \Phi^{+}(t, x) & =\sqrt{2} \delta_{x+t=0} .
\end{aligned}
$$

The two first terms of $(2.10)$ belong to $L_{l o c}^{2}$, whereas in the last one we get $\delta_{x+t=0}$.

One final comment: the first counterexample above shows that the smoothing mechanism of Velocity Averaging cannot improve upon nonresonant smoothing when condition (NR) holds ${ }^{2}$. However, the same mechanism as in Velocity Averaging helps when condition (NR) fails, if it is known that the set of $\xi$-s for which (NR) is not verified is of small measure in some sense. This situation occurs in the relativistic Vlasov-Maxwell (RVM) system when only a few particles reach large momenta. We shall explain in section 4 below how this last idea helps in studying the regularity of weak solutions of (RVM) as in [5] with smooth initial data.

\footnotetext{
${ }^{2}$ This is consistent with the fact that Velocity Averaging lemmas are used neither in the Pfaffelmoser proof of global existence of classical solutions to the Vlasov-Poisson system (see [7]), nor in the corresponding Glassey-Strauss argument for the relativistic Vlasov-Maxwell system, which both deal with solutions having bounded support in the momentum variable. Recently R. Glassey confirmed to the second author that any attempt to use the Velocity Averaging method in order to simplify the arguments in [8] or extend their validity had not been successful yet.
} 


\section{Kinetic formulation of Maxwell's equations; applica- tions to the RVM system}

By a "kinetic formulation of Maxwell's equations", we mean a representation of the four components of the electromagnetic potential as moments of a single, scalar potential which depends of course on $t$ and $x$ but also on an extra variable $\xi$. The moments mentioned above are $\xi$-averages of this potential, like $\rho_{\chi}$ in (1.3).

Maxwell's system of equations in the vacuum reads

$$
\begin{aligned}
\partial_{t} E-\nabla_{x} \wedge B & =-j, \\
\nabla_{x} \cdot E & =\rho, \\
\partial_{t} B+\nabla_{x} \wedge E & =0, \\
\nabla_{x} \cdot B & =0,
\end{aligned}
$$

where the unknowns $E \equiv E(t, x)$ and $B \equiv B(t, x)$ are respectively the electric and magnetic field, while the current $j \equiv j(t, x)$ and charge density $\rho \equiv \rho(t, x)$ are given. The system (3.1) is well-posed on $\mathbb{R}_{+} \times \mathbb{R}^{3}$ in some appropriate class of functions once initial conditions are prescribed, as follows:

$$
E_{\mid t=0}=E_{I}, \quad B_{\mid t=0}=B_{I},
$$

where $E_{I}$ and $B_{I}$ are compatible with the second and fourth equations in (3.1) and provided that $\rho$ and $j$ satisfy the continuity equation

$$
\partial_{t} \rho+\nabla_{x} \cdot j=0 .
$$

Suppose now that, instead of the macroscopic quantities $\rho$ and $j$, one is given a microscopic, phase-space density of charges $f(t, x, \xi)$, as in the kinetic theory of gases. In other words, $f(t, x, \xi)$ is the density of (like) charged particles (electrons or ions) which, at time $t$, occupy position $x$ and have momentum $\xi$. The macroscopic density of charge and the current are given in terms of the microscopic density $f$ by the formulas

$$
\rho(t, x)=\int f(t, x, \xi) d \xi, \quad j(t, x)=\int f(t, x, \xi) v(\xi) d \xi,
$$

where the velocity of particles with momentum $\xi$ is expressed as

$$
v(\xi)=\frac{\xi}{\sqrt{1+|\xi|^{2}}}
$$

in dimensionless variables. In kinetic theory, the continuity equation (3.3) is usually implied by a transport equation on $f$, of the form

$$
\partial_{t} f+v(\xi) \cdot \nabla_{x} f=S, \quad \text { with } \int S d \xi=0 .
$$


In order to satisfy (3.1)-(3.2), we first choose a vector field $A_{I} \equiv A_{I}(x)$ such that

$$
\nabla_{x} \wedge A_{I}=B_{I}, \quad \nabla_{x} \cdot A_{I}=0,
$$

and define $A^{(I)} \equiv A^{(I)}(t, x)$ by

$$
\begin{aligned}
\square_{t, x} A^{(I)} & =0, \\
A_{\mid t=0}^{(I)} & =A_{I}, \\
\partial_{t} A_{\mid t=0}^{(I)} & =-E_{I} .
\end{aligned}
$$

Solve then for $u \equiv u(t, x, \xi)$ the Cauchy problem for the wave equation

$$
\begin{aligned}
\square_{t, x} u & =f, \\
u_{\mid t=0} & =0, \\
\partial_{t} u_{\mid t=0} & =0 .
\end{aligned}
$$

Elementary computations based on the continuity equation (3.3) and the uniqueness of solutions to the Cauchy problem (3.1)-(3.2) show that

$$
\phi=\int u d \xi, \quad A=A^{(I)}+\int u v(\xi) d \xi
$$

are respectively the scalar and vector potentials satisfying the wave equations

$$
\square_{t, x} \phi=\rho, \quad \square_{t, x} A=j,
$$

the Lorentz gauge condition

$$
\partial_{t} \phi+\nabla_{x} \cdot A=0
$$

and giving the electromagnetic field by the formulas

$$
\begin{aligned}
& E=-\partial_{t} A-\nabla_{x} \phi=-\partial_{t} A^{(I)}-\partial_{t} \int u v(\xi) d \xi-\nabla_{x} \int u d \xi, \\
& B=\nabla_{x} \wedge A=\nabla_{x} \wedge A^{(I)}+\nabla_{x} \wedge \int u v(\xi) d \xi
\end{aligned}
$$

Thus Maxwell's system of equations (3.1)-(3.2) can be replaced by the single scalar wave equation (3.9) with the continuity equation implied by (3.6).

This kinetic formulation of Maxwell's system of equations is of course very natural when the electromagnetic field is the self-consistent field of a plasma. This is precisely the situation described by the relativistic Vlasov-Maxwell system. 
In this case, the source term $S$ in (3.6) is the term modeling the acceleration by the Lorentz force.

$$
\begin{aligned}
\partial_{t} f+v(\xi) \cdot \nabla_{x} f & =-(E+v(\xi) \wedge B) \cdot \nabla_{\xi} f, \\
\partial_{t} E-\nabla_{x} \wedge B & =-j_{f}, \\
\nabla_{x} \cdot E & =\rho_{f} \\
\partial_{t} B+\nabla_{x} \wedge E & =0 \\
\nabla_{x} \cdot B & =0
\end{aligned}
$$

with $v(\xi)$ as in (3.5) and the notations

$$
\rho_{f}=\int f(t, x, \xi) d \xi, \quad j_{f}=\int f(t, x, \xi) v(\xi) d \xi
$$

This system for the unknown $(f, E, B) \equiv(f(t, x, \xi), E(t, x), B(t, x))$ is posed in $\mathbb{R}_{+} \times \mathbb{R}_{x}^{3} \times \mathbb{R}_{\xi}^{3}$ and is completed by the initial conditions

$$
f_{\mid t=0}=f_{I}, \quad E_{\mid t=0}=E_{I}, \quad B_{\mid t=0}=B_{I} .
$$

The main results known to this date on (RVM) are

- the global existence of weak (and even renormalized) solutions, proved by R. DiPerna and P.-L. Lions [5];

- existence and uniqueness of classical solutions under the assumption that $\operatorname{supp} f(t, x, \cdot)$ is bounded for each $t>0$, proved by R. Glassey and W. Strauss [8].

Subsequently, the global existence and uniqueness of classical solutions to (RVM) was established in [9] under the weaker assumption that the macroscopic energy density satisfy

$$
\int \sqrt{1+|\xi|^{2}} f d \xi \in L_{l o c}^{\infty}\left(\mathbb{R}_{+} ; L^{\infty}\left(\mathbb{R}^{3}\right)\right) .
$$

Finally, R. Glassey and W. Strauss established the global existence and uniqueness of classical solutions to (RVM) for small (in some sense) initial data in [10], by proving that (3.16) holds for such initial data.

The main open problem on (RVM) is to prove (or disprove) the same result as in [8] without assuming (3.16) or the support condition for all $t>0$ :

"Let $f_{I}, E_{I}$ and $B_{I}$ be compactly supported and $C^{\infty}$. Does there exist a unique global $C^{\infty}$ solution to the Cauchy problem (3.13)-(3.15)?"

The system (RVM) can be somewhat simplified by using the kinetic formulation of the Maxwell equation. It becomes

$$
\begin{aligned}
\partial_{t} f+v(\xi) \cdot \nabla_{x} f & =\nabla_{\xi} \cdot[-(E+v(\xi) \wedge B) f], \\
\square_{t, x} u & =f,
\end{aligned}
$$


where $(E, B)$ are given in terms of $u$ by (3.12). The initial conditions are

$$
f_{\mid t=0}=f_{I}, \quad u_{\mid t=0}=\partial_{t} u_{\mid t=0}=0 .
$$

The formulation (3.17)-(3.18)-(3.12) of (RVM) is the main reason for considering coupled wave + transport systems as (1.1). It greatly simplifies the formulas in [8] representing the electromagnetic field in terms of the acceleration part in the transport equation of (RVM). Indeed, these formulas occupy 13 of the 32 pages in [8] and their complexity somewhat hinders a complete understanding of the key arguments in this otherwise carefully written paper.

Finally, let us mention that the functions $u$ and $v(\xi) u$ with $u$ as in (3.17) are natural physical quantities. They can be viewed as the Liénard-Wiechert potentials (see $[16, \S 63]$ ) distributed under the initial microscopic density $f_{I}$.

\section{Regularity of solutions of the (RVM) system}

This section expands on the idea introduced in the last paragraph of section 2, namely merging the techniques of Velocity Averaging with nonresonant smoothing. We concentrate on the important example of the (RVM) system, for which we have been able to establish the following a priori regularity result on the electromagnetic field.

Theorem 2 Consider initial data $\left(f_{I}, E_{I}, B_{I}\right)$ such that $f_{I} \in L^{\infty}\left(\mathbb{R}^{3} \times \mathbb{R}^{3}\right)$, $f_{I} \geq 0$ a.e., $E_{I}$ and $B_{I} \in H_{l o c}^{1}\left(\mathbb{R}^{3}\right)$ satisfy

$$
\nabla_{x} \cdot B_{I}=0, \quad \nabla_{x} \cdot E_{I}=\int f_{I} d \xi
$$

and the finite energy condition

$$
\iint \sqrt{1+|\xi|^{2}} f_{I} d x d \xi+\int\left(\left|E_{I}\right|^{2}+\left|B_{I}\right|^{2}\right) d x<+\infty
$$

holds. Let $(f, E, B)$ be a weak solution of the (RVM) system (the existence of which is predicted by [5]). If the macroscopic energy density satisfies

$$
\left.\left.\int \sqrt{1+|\xi|^{2}} f d \xi \in L_{l o c}^{p}\left(\mathbb{R}_{+} \times \mathbb{R}^{3}\right), \quad \text { with } p \in\right] \frac{3}{2}, 2\right]
$$

then the electromagnetic field has regularity given by

$$
E \text { and } B \in H_{l o c}^{s}\left(\mathbb{R}_{+}^{*} \times \mathbb{R}^{3}\right), \quad \text { with } s<\frac{4 p-6}{4 p+3} \text {. }
$$


Before giving the proof of this result, let us stress a few points. Observe first that the condition (4.3) is indeed weaker than the condition (3.16) under which R. Glassey and W. Strauss have proved in [9] the global existence and uniqueness of a classical solution, with

$$
E \text { and } B \text { belonging to } L_{\text {loc }}^{\infty}\left(\mathbb{R}_{+} ; W^{1, \infty}\left(\mathbb{R}^{3}\right)\right) \text {. }
$$

Accordingly, the regularity on $E$ and $B$ predicted by theorem 2 is weaker. Besides, taking $p=\infty$ and copying the proof of theorem 2 would not give the $W^{1, \infty}$ control of [9], which is based on iterating twice a rather intricate procedure which is close in spirit to the mechanism of nonresonant smoothing described above. It would instead give a weaker piece of information, namely that $E$ and $B \in H_{l o c}^{1}\left(\mathbb{R}_{+} \times \mathbb{R}^{3}\right)$. It could be that the regularity predicted in theorem 2 is not optimal and would be improved by using part of the information in [8] or [9].

However, the assumption (4.3) is not more natural than (3.16). The only natural condition on the macroscopic energy density is that

$$
\int \sqrt{1+|\xi|^{2}} f(t, x, \xi) d \xi \in L_{t}^{\infty}\left(L_{x}^{1}\right)
$$

which is guaranteed ${ }^{3}$ by the conservation of energy for (RVM) but we have not yet been able to use it to control the density of particles with large momenta in a way compatible with nonresonant smoothing as suggested in the last paragraph of section 2 .

Our second main observation on theorem 2 is that the Sobolev regularity index it predicts exceeds that predicted by Velocity Averaging. For example, in the case where (4.3) holds with $p=2$, a direct application of the Velocity Averaging lemma of [5] (or Theorem 1.5.6 of [3]) would imply that

$$
\rho=\int f d \xi \text { and } j=\int v(\xi) f d \xi \in H_{l o c}^{1 / 16}\left(\mathbb{R}_{+}^{*} \times \mathbb{R}^{3}\right)
$$

which, by the classical energy estimate for Maxwell's system, entails that

$$
E \text { and } B \in H_{l o c}^{1 / 16}\left(\mathbb{R}_{+}^{*} \times \mathbb{R}^{3}\right) .
$$

This regularity is indeed weaker than the one predicted by theorem 2 , in this case that

$$
E \text { and } B \in H_{l o c}^{2 / 11}\left(\mathbb{R}_{+}^{*} \times \mathbb{R}^{3}\right) .
$$

At variance with the Velocity Averaging method however, theorem 2 says nothing of the regularity of the density of charge $\rho$ and current $j$.

\footnotetext{
${ }^{3}$ Actually, the theory of weak solutions to the (RVM) only predicts that the total energy at time $t$ is less than or equal to that at time 0 , for any positive $t$.
} 
Proof of Theorem 2. First, a simple interpolation argument leads to $L^{2}$ estimates on the charge and current densities.

Lemma 4 Let $f \equiv f(t, x, \xi)$ be a measurable function on $\mathbb{R}_{+} \times \mathbb{R}^{3} \times \mathbb{R}^{3}$. Then, for each $\alpha \in[0,1]$, one has

$$
\left\|\int|f| d \xi\right\|_{L_{t, x}^{2}} \leq 9\|f\|_{L_{t, x, \xi}^{\infty+3}}^{\frac{\alpha}{\alpha+3}}\left\|\int|\xi|^{\alpha}|f| d \xi\right\|_{L_{t, x}^{\frac{6}{\alpha+3}}}^{\frac{3}{\alpha+3}} .
$$

Further, for each $R>0$

$$
\left\|\int_{|\xi|>R}|f| d \xi\right\|_{L_{t, x}^{2}} \leq \frac{9}{R^{\frac{3(1-\alpha)}{\alpha+3}}}\|f\|_{L_{t, x, \xi}^{\infty}}^{\frac{\alpha}{\alpha+3}}\left\|\int \sqrt{1+|\xi|^{2}}|f| d \xi\right\|_{L_{t, x}^{\frac{6}{\alpha+3}}}^{\frac{3}{\alpha+3}} .
$$

Proof of Lemma 4. We have for each $R>0$ :

$$
\begin{aligned}
& \int|f| d \xi=\int_{|\xi| \leq R}|f| d \xi+\int_{|\xi|>R}|f| d \xi \\
& \int|f| d \xi \leq \frac{4 \pi}{3} R^{3}\|f\|_{L_{t, x, \xi}^{\infty}}+\frac{1}{R^{\alpha}} \int|\xi|^{\alpha}|f| d \xi .
\end{aligned}
$$

Taking $R$ such that

$$
R^{3}\|f\|_{L_{t, x, \xi}^{\infty}}=\frac{1}{R^{\alpha}} \int|\xi|^{\alpha}|f| d \xi
$$

and since $\frac{4 \pi}{3} \leq 8$, it comes

$$
\int|f| d \xi \leq 9\|f\|_{L_{t, x, \xi}^{\infty}}^{\frac{\alpha}{\alpha+3}}\left(\int|\xi|^{\alpha}|f| d \xi\right)^{\frac{3}{\alpha+3}} .
$$

The estimate (4.7) is obtained from (4.6) applied to the function $11_{|\xi|>R} f$ :

$$
\begin{aligned}
& \left\|\int_{|\xi|>R}|f| d \xi\right\|_{L_{t, x}^{2}} \leq 9\|f\|_{L_{t, x, \xi}^{\infty+3}}^{\frac{\alpha}{\alpha+3}}\left\|\int_{|\xi|>R}|\xi|^{\alpha}|f| d \xi\right\|_{L^{\frac{6}{\alpha+3}}}^{\frac{3}{\alpha+3}}, \\
& \left\|\int_{|\xi|>R}|f| d \xi\right\|_{L_{t, x}^{2}} \leq \frac{9}{R^{\frac{3(1-\alpha)}{\alpha+3}}}\|f\|_{L_{t, x, \xi}^{\infty}}^{\frac{\alpha}{\alpha+3}}\left\|\int_{|\xi|>R}|\xi||f| d \xi\right\|_{L^{\frac{6}{\alpha+3}}}^{\frac{3}{\alpha+3}}, \\
& \left\|\int_{|\xi|>R}|f| d \xi\right\|_{L_{t, x}^{2}} \leq \frac{9}{R^{\frac{3(1-\alpha)}{\alpha+3}}}\|f\|_{L_{t, x, \xi}^{\infty}}^{\frac{\alpha}{\alpha+3}}\left\|\int \sqrt{1+|\xi|^{2}}|f| d \xi\right\|_{L^{\frac{6}{\alpha+3}}}^{\frac{3}{\alpha+3}} .
\end{aligned}
$$

The second step in the proof of theorem 2 is a more accurate version of lemma 1. 
Lemma 5 Let $v \equiv v(\xi) \in W^{1, \infty}\left(\mathbb{R}^{3}\right)$ satisfy $|v(\xi)|<1$ for all $\xi \in \mathbb{R}^{3}$. For each $\lambda \in]|v(\xi)|^{2}, 1\left[\right.$, set $q_{\xi}^{\lambda}(\omega, k)=\omega^{2}-|v(\xi) \cdot k|^{2}-\lambda\left(\omega^{2}-|k|^{2}\right)$. Then, for each $\xi \in \mathbb{R}^{3}$,

$$
\inf _{|v(\xi)|^{2}<\lambda<1} \sup _{\omega^{2}+|k|^{2}>0} \frac{\omega^{2}+|k|^{2}}{q_{\xi}^{\lambda}(\omega, k)}=\frac{2}{1-|v(\xi)|^{2}},
$$

with the inf attained at $\lambda(\xi)=\frac{1}{2}\left(1+|v(\xi)|^{2}\right)$. For such choice of $\lambda$,

$$
\sup _{\omega^{2}+|k|^{2}>0}\left(\omega^{2}+|k|^{2}\right)\left|D_{\xi} \frac{1}{q_{\xi}^{\lambda}(\omega, k)}\right| \leq \frac{12|\nabla v(\xi)|}{\left(1-|v(\xi)|^{2}\right)^{2}} .
$$

Proof of Lemma 5. We write the Cauchy-Schwarz inequality for $q_{\xi}^{\lambda}$ :

$$
q_{\xi}^{\lambda}(\omega, k) \geq(1-\lambda) \omega^{2}+\left(\lambda-|v(\xi)|^{2}\right)|k|^{2},
$$

This becomes an equality if $v(\xi)$ and $k$ are linearly dependent, so that

$$
\begin{aligned}
\sup _{\omega^{2}+|k|^{2}>0} \frac{\omega^{2}+|k|^{2}}{q_{\xi}^{\lambda}(\omega, k)} & =\sup _{\omega^{2}+|k|^{2}>0} \frac{\omega^{2}+|k|^{2}}{(1-\lambda) \omega^{2}+\left(\lambda-|v(\xi)|^{2}\right)|k|^{2}} \\
& =\sup _{(r, \theta) \in \mathbb{R}_{+}^{*} \times[0,2 \pi[} \frac{r^{2}}{(1-\lambda) r^{2} \cos ^{2} \theta+\left(\lambda-|v(\xi)|^{2}\right) r^{2} \sin ^{2} \theta} \\
& =\sup _{\theta \in[0,2 \pi[} \frac{1}{(1-\lambda) \cos ^{2} \theta+\left(\lambda-|v(\xi)|^{2}\right) \sin ^{2} \theta} \\
& =\max \left(\frac{1}{1-\lambda}, \frac{1}{\lambda-|v(\xi)|^{2}}\right) .
\end{aligned}
$$

The functions

$$
\lambda \mapsto \frac{1}{1-\lambda} \quad \text { and } \quad \lambda \mapsto \frac{1}{\lambda-|v(\xi)|^{2}}
$$

defined for $\lambda \in]|v(\xi)|^{2}, 1[$ are nondecreasing and nonincreasing respectively so the lower bound for the right hand side of (4.10) is attained at $\lambda$ such that the equality

$$
\frac{1}{1-\lambda}=\frac{1}{\lambda-|v(\xi)|^{2}}
$$

holds. This implies

$$
\lambda=\frac{1}{2}\left(1+|v(\xi)|^{2}\right)
$$

and gives the bound (4.8). 
Now we establish (4.9):

$$
\begin{aligned}
\left(\omega^{2}+|k|^{2}\right)\left|D_{\xi} \frac{1}{q_{\xi}^{\lambda}(\omega, k)}\right| & =\left(\omega^{2}+|k|^{2}\right) \frac{\left(D_{\xi} v \cdot v\right)\left(\omega^{2}-|k|^{2}\right)+2\left(D_{\xi} v \cdot k\right)(v(\xi) \cdot k)}{q_{\xi}^{\lambda}(\omega, k)^{2}} \\
& \leq\left(\omega^{2}+|k|^{2}\right) \frac{3|\nabla v(\xi)|\left(|k|^{2}+\omega^{2}\right)}{q_{\xi}^{\lambda}(\omega, k)^{2}} \\
& \leq 3|\nabla v(\xi)|\left(\frac{\omega^{2}+|k|^{2}}{q_{\xi}^{\lambda}(\omega, k)}\right)^{2},
\end{aligned}
$$

where we used

$$
\lambda(\xi)=\frac{1}{2}\left(1+|v(\xi)|^{2}\right)
$$

and the assumptions $v \in W^{1, \infty}$ and $|v|<1$. We conclude that

$$
\sup _{\omega^{2}+|k|^{2}>0}\left(\omega^{2}+|k|^{2}\right)\left|D_{\xi} \frac{1}{q_{\xi}^{\lambda}(\omega, k)}\right| \leq 3|\nabla v(\xi)| \frac{4}{\left(1-|v(\xi)|^{2}\right)^{2}} .
$$

With these estimates at our disposal, the proof of theorem 2 follows the line of the Velocity Averaging method. The main idea, as can be seen in [11], [12] and [5], consists in splitting the momentum space in two regions:

- in the first region, defined by the inequality $|\xi| \leq R$, the speed of particles is bounded by

$$
|v(\xi)| \leq \frac{R}{\sqrt{1+R^{2}}} ;
$$

hence the condition (NR) is satisfied so that nonresonant smoothing holds for the electromagnetic field created by these particles; further, the ellipticity estimates (4.8) and (4.9) control the growth of the $H^{1}$ norm of this part of the electromagnetic field as $R \rightarrow+\infty$;

- in the second region, defined by the inequality $|\xi|>R$, the control on the macroscopic energy density (4.3) together with the estimates (4.6) and (4.7) imply that the densities of charge and current created by the corresponding particles are small in $L^{2}$ as $R \rightarrow+\infty$; the $L^{2}$ norms of the corresponding fields are then controlled by the classical energy estimate for Maxwell's system of equations.

Let $\Theta_{R} \in C_{c}^{\infty}\left(\mathbb{R}^{3}\right)$ be a cut-off function verifying

$$
\begin{array}{ll}
\Theta_{R}(\xi)=1 \quad \forall|\xi| \leq R \\
\Theta_{R}(\xi)=0 \quad \forall|\xi|>2 R .
\end{array}
$$


We define

$$
\begin{aligned}
& u_{1}=\theta_{R} u, \quad u_{2}=\left(1-\theta_{R}\right) u, \\
& f_{1}=\theta_{R} f, \quad f_{2}=\left(1-\theta_{R}\right) f,
\end{aligned}
$$

so that

$$
u=u_{1}+u_{2} \quad \text { and } \quad f=f_{1}+f_{2},
$$

with the two systems:

$$
\begin{aligned}
\square_{t, x} u_{1} & =f_{1}, \\
\partial_{t} f_{1}+v \cdot \nabla_{x} f_{1} & =-\Theta_{R} \nabla_{\xi} \cdot[(E+v \wedge B) f], \\
\square_{t, x} u_{2} & =f_{2}, \\
\partial_{t} f_{2}+v \cdot \nabla_{x} f_{2} & =-\left(1-\Theta_{R}\right) \nabla_{\xi} \cdot[(E+v \wedge B) f],
\end{aligned}
$$

with initial conditions:

$$
\begin{gathered}
u_{1 \mid t=0}=0, \quad \partial_{t} u_{1 \mid t=0}=0, \quad f_{1 \mid t=0}=\Theta_{R} f_{I}, \\
u_{2 \mid t=0}=0, \quad \partial_{t} u_{2 \mid t=0}=0, \quad f_{2 \mid t=0}=\left(1-\Theta_{R}\right) f_{I} .
\end{gathered}
$$

Recall that the fields are given by:

$$
\begin{aligned}
& E=-\partial_{t} A^{I}-\partial_{t} \int u v(\xi) d \xi-\nabla_{x} \int u d \xi \\
& B=\nabla_{x} \wedge A^{I}+\nabla_{x} \wedge \int u v(\xi) d \xi
\end{aligned}
$$

We recast these expressions as:

$$
\begin{aligned}
E & =-\partial_{t} A^{I}-\int\left(\partial_{t} u_{1} v(\xi)+\nabla_{x} u_{1}\right) d \xi-\int\left(\partial_{t} u_{2} v(\xi)+\nabla_{x} u_{2}\right) d \xi \\
& =E^{I}+E_{1}+E_{2}, \\
B & =\nabla_{x} \wedge A^{I}+\int \nabla_{x} \wedge u_{1} v(\xi) d \xi+\int \nabla_{x} \wedge u_{1} v(\xi) d \xi \\
& =B^{I}+B_{1}+B_{2} .
\end{aligned}
$$

The part dealing with initial data $E^{I}$ and $B^{I}$ has the desired smoothness.

We consider now $E_{1}$ and $B_{1}$ for which we can use nonresonant smoothing. For any test function $\psi \in C_{c}^{\infty}\left(\mathbb{R}_{+} \times \mathbb{R}^{3}\right)$, theorem 1 applied to (4.11) gives:

$$
\begin{gathered}
\left\|\int \psi u_{1} \Theta_{2 R}(\xi) d \xi\right\|_{H^{2}} \leq C_{\psi, R}\|f\|_{L^{2}\left(K_{\psi}\right)}+C_{\psi, R}\|(E+v \wedge B) f\|_{L^{2}\left(K_{\psi}\right)}, \\
\left\|\int \psi u_{1} v(\xi) \Theta_{2 R}(\xi) d \xi\right\|_{H^{2}} \leq C_{\psi, R}^{\prime}\|f\|_{L^{2}\left(K_{\psi}\right)}+C_{\psi, R}^{\prime}\|(E+v \wedge B) f\|_{L^{2}\left(K_{\psi}\right)} .
\end{gathered}
$$


The constants $C_{\psi, R}$ and $C_{\psi, R}^{\prime}$ depend on $R$. The growth with respect to $R$ is read in (2.7) and (2.9). We use lemma 5 to bound it:

$$
\left\|\int\left[\left(\omega^{2}+|k|^{2}\right) \frac{\Theta_{2 R}}{q_{\xi}^{\lambda}}\right]^{2} d \xi\right\|_{L_{\omega, k}^{\infty}}^{\frac{1}{2}} \leq 2\left(\int_{|\xi|<4 R}\left(1+|\xi|^{2}\right)^{2} d \xi\right)^{\frac{1}{2}} \leq C R^{\frac{7}{2}}
$$

and

$$
\begin{aligned}
\left\|\int\left[\left(\omega^{2}+|k|^{2}\right) \Theta_{2 R} D_{\xi}\left(\frac{1}{q_{\xi}^{\lambda}}\right)\right]^{2} d \xi\right\|_{L_{\omega, k}^{\infty}}^{\frac{1}{2}} & \leq 12\left(\int_{|\xi|<4 R}|\nabla v(\xi)|^{2}\left(1+|\xi|^{2}\right)^{4} d \xi\right)^{\frac{1}{2}} \\
& \leq C R^{\frac{9}{2}}
\end{aligned}
$$

We infer that:

$$
\max \left(C_{\psi, R}, C_{\psi, R}^{\prime}\right) \leq C_{\psi} R^{9 / 2} .
$$

Moreover the following estimates are given by [3]:

$$
\|f(t)\|_{L_{x, \xi}^{\infty}} \leq\left\|f_{I}\right\|_{L_{x, \xi}^{\infty}}
$$

and

$$
\iint|\xi| f(t, x, \xi) d x d \xi+\int|E(t, x)|^{2}+|B(t, x)|^{2} d x \leq C<+\infty .
$$

Hence we get:

$$
\left\|\psi E_{1}\right\|_{H^{1}}+\left\|\psi B_{1}\right\|_{H^{1}} \leq C R^{\frac{9}{2}} .
$$

Next we estimate $E_{2}$ and $B_{2}$. Let $\chi \in C_{c}^{\infty}\left(\mathbb{R}_{+} \times \mathbb{R}^{3}\right)$ verifying $|\chi| \leq 1$.

We use (4.7) with $\chi f v$ and $\chi f$ :

$$
\left\|\int_{|\xi|>R}|\chi f v| d \xi\right\|_{L_{t, x}^{2}} \leq \frac{9}{R^{\frac{3(1-\alpha)}{\alpha+3}}}\|\chi f v\|_{L_{t, x, \xi}^{\infty}}^{\frac{\alpha}{\alpha+3}}\left\|\int \sqrt{1+|\xi|^{2}}|\chi f v| d \xi\right\|_{L^{\frac{6}{\alpha+3}}}^{\frac{3}{\alpha+3}}
$$

and

$$
\left\|\int_{|\xi|>R}|\chi f| d \xi\right\|_{L_{t, x}^{2}} \leq \frac{9}{R^{\frac{3(1-\alpha)}{\alpha+3}}}\|\chi f\|_{L_{t, x, \xi}^{\infty+3}}^{\frac{\alpha}{\alpha+3}}\left\|\int \sqrt{1+|\xi|^{2}}|\chi f| d \xi\right\|_{L^{\frac{6}{\alpha+3}}}^{\frac{3}{\alpha+3}}
$$

Define

$$
j_{>R} \equiv \int_{|\xi|>R} f v d \xi
$$

and

$$
\rho_{>R} \equiv \int_{|\xi|>R} f d \xi
$$

the current density and charge density created by high energy particles. 
The previous inequalities become:

$$
\begin{aligned}
\left\|\chi j_{>R}\right\|_{L_{t, x}^{2}} & \leq \frac{9}{R^{\frac{3(1-\alpha)}{\alpha+3}}}\|f\|_{L_{t, x, \xi}^{\alpha+3}}^{\frac{\alpha}{\alpha+3}}\left\|\int \sqrt{1+|\xi|^{2}}|\chi f| d \xi\right\|_{L^{\frac{6}{\alpha+3}}}^{\frac{3}{\alpha+3}}, \\
\left\|\chi \rho_{>R}\right\|_{L_{t, x}^{2}} & \leq \frac{9}{R^{\frac{3(1-\alpha)}{\alpha+3}}}\|f\|_{L_{t, x, \xi}^{\alpha+3}}^{\frac{\alpha}{\alpha+3}}\left\|\int \sqrt{1+|\xi|^{2}}|\chi f| d \xi\right\|_{L^{\frac{6}{\alpha+3}}}^{\frac{3}{\alpha+3}},
\end{aligned}
$$

where we used $|\chi| \leq 1$ and $|v| \leq 1$. But we assumed that:

$$
\left.\left.\int \sqrt{1+|\xi|^{2}} f d \xi \in L_{l o c}^{p}\left(\mathbb{R}_{+} \times \mathbb{R}^{3}\right) \quad \text { with } p \in\right] \frac{3}{2}, 2\right] .
$$

So fix $\alpha$ such that

$$
p=\frac{6}{\alpha+3} .
$$

Since (4.15) ensures $f \in L_{t, x, \xi}^{\infty}$ it follows:

$$
\exists C_{\alpha} \quad \max \left(\left\|\chi \rho_{>R}\right\|_{L_{t, x}^{2}},\left\|\chi j_{>R}\right\|_{L_{t, x}^{2}}\right) \leq 9 C_{\alpha} R^{-\frac{3(1-\alpha)}{\alpha+3}}=9 C_{\alpha} R^{3-2 p} .
$$

Now we use a local energy inequality for the wave equation:

Lemma 6 Let $\Omega \subset \mathbb{R}^{n}, f \in L^{1}\left([0, T], L^{2}(\Omega)\right)$ with $T>0$, and consider the solution $u$ to the Cauchy problem for the wave equation:

$$
\begin{aligned}
\square_{t, x} u & =f, \\
u_{\mid t=0} & =0, \\
\partial_{t} u_{\mid t=0} & =0 .
\end{aligned}
$$

Pick $x_{0} \in \Omega, r \geqslant T$ such that

$$
B_{r} \equiv\left\{x \in \mathbb{R}^{n}:\left|x-x_{0}\right| \leq r\right\} \subset \Omega .
$$

Define for any $t \in[0, T]$

$$
\mathcal{E}(t)=\int_{B_{r-t}}\left|\partial_{t} u(t, x)\right|^{2}+\left|\nabla_{x} u(t, x)\right|^{2} d x .
$$

Then the following estimate holds:

$$
\mathcal{E}(T)^{\frac{1}{2}} \leq \int_{0}^{T}\left(\int_{B_{r-t}}|f(t, x)|^{2} d x\right)^{\frac{1}{2}} d t .
$$


We apply lemma 6 to $\int u_{2} d \xi$ which solves the equation:

$$
\square_{t, x} \int u_{2} d \xi=\int f_{2} d \xi
$$

with null initial data. Then pick $\chi, r$ and $T$ such that:

$$
\operatorname{Supp} \psi \subset\left\{(t, x) \in[0, T] \times \mathbb{R}^{3}: t+|x| \leq r\right\} \subset \chi^{-1}(\{1\}) .
$$

We get the inequality

$$
\int_{B_{r-T}}\left|\partial_{t} \int u_{2} d \xi\right|^{2}+\left|\nabla_{x} \int u_{2} d \xi\right|^{2} d x \leq\left(\int_{0}^{T}\left(\int_{B_{r-t}}\left|\int f_{2} d \xi\right|^{2} d x\right)^{\frac{1}{2}} d t\right)^{2} .
$$

With the assumptions on supports, the right hand side is bounded by:

$$
T\left\|\chi \int f_{2} d \xi\right\|_{L_{x}^{2}}^{2} \leq T\left\|\chi \rho_{>R}\right\|_{L_{x}^{2}}^{2}
$$

We have also a lower bound for the left hand side:

$$
\frac{1}{\|\psi\|_{\infty}^{2}}\left(\left\|\psi \partial_{t} \int u_{2} d \xi\right\|_{L_{x}^{2}}^{2}+\left\|\psi \nabla_{x} \int u_{2} d \xi\right\|_{L_{x}^{2}}^{2}\right)
$$

Gathering these two parts, we obtain estimates for the derivatives of $\int u_{2} d \xi$ :

$$
\max \left(\left\|\psi \partial_{t} \int u_{2} d \xi\right\|_{L_{t, x}^{2}},\left\|\psi \nabla_{x} \int u_{2} d \xi\right\|_{L_{t, x}^{2}}\right) \leq C R^{3-2 p}
$$

We can also apply lemma 6 to $\int u_{2} v d \xi$. We then get the same bound. Estimates for the electromagnetic field follow:

$$
\left\|\psi E_{2}\right\|_{L_{t, x}^{2}} \leq C_{\psi} R^{3-2 p},
$$

and

$$
\left\|\psi B_{2}\right\|_{L_{t, x}^{2}} \leq C_{\psi}^{\prime} R^{3-2 p} .
$$

Hence the electromagnetic field can be split as the sum of a field whose $H^{1}$ norm tends to infinity with $R$ and of a field whose $L^{2}$ norm vanishes with $1 / R$. One concludes by a straightforward interpolation argument; $\psi E$ and $\psi B$ belong to $H^{s}$ whenever $s<\theta$, with

$$
\theta=\frac{2 p-3}{2 p-3+\frac{9}{2}}=\frac{4 p-6}{4 p+3}
$$




\section{Conclusion}

In this note, we have discussed a mechanism of nonresonant smoothing for a wave equation coupled to a transport equation. The main application of this idea seems to be the (RVM) system, with Maxwell's system of equations reduced to a scalar wave equation by a kinetic formulation reminiscent of the notion of Liénard-Wiechert potentials. While theorem 2 exemplifies the power of using the method of Velocity Averaging together with nonresonant smoothing, it is very likely that the regularity statement (4.4) is not optimal.

However, we believe that the idea of using (some form of) nonresonant smoothing for the vast majority of particles with momenta below a certain threshold together with the method of Velocity Averaging to control the few particles with higher momenta might prove helpful in the question of global existence of classical solutions to the (RVM) system, still open to this date except for special cases (small data, almost neutral initial data, $2+1 / 2 D$ solutions... )

Finally, this mechanism of nonresonant smoothing is by no means confined to the examples considered here, but can easily be generalized to a wide generality of systems of coupled hyperbolic equations.

For example, one could think of models arising in the study of the laser-plasma interaction or Langmuir turbulence. Such models involve a wave equation for the electromagetic field and another wave equation for the acoustic disturbances in the plasma coupled by terms involving in particular the ponderomotive force: see [1], or the contribution by D. Pesme in [4].

The nonresonance condition adapted to a system of two coupled wave equations reduces to saying that the speeds of propagation in both wave equations are different, a condition obviously satisfied in the case of laserplasma interaction, where the speed of light is to be compared to the speed of sound in the plasma. In this case, nonresonant smoothing entails a gain of three derivatives on one of the fields, which might be useful in the mathematical treatment of models such as considered in [1].

Note added in proof. S. Klainerman recently informed the second author of a new approach to the Glassey-Strauss theorem by himself and G. Staffilani [15]. One of the steps in their proof is similar to the integration by parts in $\xi$ using estimate (2.3). 


\section{References}

[1] Bergé, L., Bidégaray, B. And Colin, T.: A perturbative analysis of the time-envelope approximation in strong Langmuir turbulence. Phys. D 95 (1996), 351-379.

[2] Bogoliubov, N. N. And Mitropolsky, Y.A.: Asymptotic methods in the theory of non-linear oscillations. Translated from the second revised Russian edition. International Monographs on Advanced Mathematics and Physics. Hindustan Publishing Corp., Delhi, Gordon and Breach Science Publishers, New York 1961.

[3] Bouchut, F., Golse, F. And Pulvirenti, M.: Kinetic Equations and Asymptotic Theory. Series in Applied Mathematics 4. Gauthier-Villars, Editions Scientifiques et Médicales Elsevier, Paris; North-Holland, Amsterdam, 2000.

[4] Dautray, R. and Watteau, J.: La fusion thermonucléaire inertielle par laser. Eyrolles, Paris 1993.

[5] DiPerna, R. And Lions, P.-L.: Global weak solutions of Vlasov-Maxwell systems. Comm. Pure Appl. Math. 42 (1989), no. 6, 729-757.

[6] Fefferman, C. and Stein, E. M.: $H^{p}$ spaces of several variables. Acta Math. 129 (1972), no. 3-4, 137-193.

[7] Glassey, R. T.: The Cauchy problem in kinetic theory. Society for Industrial and Applied Mathematics (SIAM), Philadelphia, PA, 1996.

[8] Glassey, R. T. and Strauss, W. A.: Singularity formation in a collisionless plasma could occur only at high velocities. Arch. Rational Mech. Anal. 92 (1986), no. 1, 59-90.

[9] Glassey, R. T. and Strauss, W. A.: High Velocity Particles in a Collisionless Plasma. Math. Meth. Appl. Sci. 9 (1987), 46-52.

[10] Glassey, R. T. and Strauss, W. A.: Absence of schocks in an initially dilute collisionless plasma. Comm. Math. Phys. 113 (1987), 191-208.

[11] Golse, F., Perthame, B. And Sentis, R.: Un résultat de compacité pour les équations de transport et application au calcul de la valeur propre principale d'un opérateur de transport. C. R. Acad. Sci. Paris Sér. I Math. 301 (1985), 341-344.

[12] Golse, F., Lions, P.-L., Perthame, B. and Sentis, R.: Regularity of the moments of the solution of a transport equation. J. Funct. Anal. 76 (1988), 110-125.

[13] Hörmander, L.: Lectures on nonlinear hyperbolic differential equations. Mathématiques and Applications 26. Springer-Verlag, Berlin, 1997.

[14] Klainerman, S. and Machedon, M.: Finite energy solutions of the Yang-Mills equations in $\mathbb{R}^{3+1}$. Ann. of Math. (2) 142 (1995), 39-119.

[15] Klainerman, S. and Staffilani, G.: A new approach to study the Vlasov-Maxwell system. Commun. Pure Appl. Anal. 1 (2002), 103-125. 
[16] Landau, L. And Lifshitz, E.: Cours de physique théorique. Vol. 2: Théorie des champs. Editions Mir, Moscou, 1970.

[17] Shatah, J. and Struwe, M.: Geometric wave equations. Courant Lecture Notes in Mathematics 2. New York University, Courant Institute of Mathematical Sciences, New York. American Mathematical Society, Providence, RI, 1998.

Recibido: 5 de septiembre de 2002

François Bouchut

C.N.R.S. and D.M.A.

Ecole Normale Supérieure

45 rue d'Ulm

75230 Paris cedex 05, France

fbouchut@dma.ens.fr

François Golse

Laboratoire J.-L. Lions

Université Paris 7

Boîte courrier 187

75252 Paris cedex 05, France

golse@math.jussieu.fr

Christophe Pallard

D.M.A.

Ecole Normale Supérieure

45 rue d'Ulm

75230 Paris cedex 05, France

pallard@dma.ens.fr

This work is partially supported by the European T.M.R. network "Asymptotic methods in kinetic theory", contract no. ERB- FMRXCT970157. 